\title{
Prediction of 24-hour sodium excretion from spot urine samples in South African adults: a comparison of four equations
}

\author{
Karen Charlton $\mathbb{1}^{1} \cdot$ Lisa J. Ware ${ }^{2}$ Glory Chidumwa $\mathbb{C}^{2} \cdot$ Marike Cockeran $^{3} \cdot$ Aletta E. Schutte $\mathbb{C}^{4,5} \cdot$ \\ Nirmala Naidoo ${ }^{6}$ Paul Kowal $\mathbb{D}^{6,7,8}$
}

Received: 7 January 2019 / Revised: 22 February 2019 / Accepted: 13 March 2019 / Published online: 10 May 2019

(c) Springer Nature Limited 2019

\begin{abstract}
Repeated 24-hour urine collection is considered to be the gold standard for assessing salt intake. This is often impractical in large-population studies, especially in low-middle-income countries. Equations to estimate 24-hour urinary salt excretion from a spot urine sample have been developed, but have not been widely validated in African populations. This study aimed to systematically assess the validity of four existing equations to predict 24-hour urinary sodium excretion (24UNa) from spot urine samples in a nationally representative sample of South Africans. Spot and 24-hour urine samples were collected in a subsample $(n=438)$ of participants from the World Health Organisation Study on global AGEing and adult health (SAGE) Wave 2 in South Africa in 2015. Measured 24UNa values were compared with predicted 24UNa values from the Kawasaki, Tanaka, INTERSALT and Mage equations using Bland-Altman plots. In this subsample (mean age $52.8 \pm$ 16.4 years; body mass index $30.2 \pm 8.2 \mathrm{~kg} / \mathrm{m}^{2} ; 76 \%$ female; $73 \%$ black African; $42 \%$ hypertensive), all four equations produced a significantly different population estimate compared with the measured median value of $6.7 \mathrm{~g}$ salt/day (IQR 4.4-10.5). Although INTERSALT underestimated salt intake $(-3.77 \mathrm{~g} / \mathrm{d} ;-1.64$ to -7.09$)$, the other equations overestimated by $1.28 \mathrm{~g} / \mathrm{d}(-3.52 ; 1.97), 6.24 \mathrm{~g} / \mathrm{d}(2.22 ; 9.45)$, and $17.18 \mathrm{~g} / \mathrm{d}(8.42 ; 31.96)$ for Tanaka, Kawasaki, and Mage, respectively. Bland-Altman curves indicated unacceptably wide levels of agreement. Use of these equations to estimate population level salt intake from spot urine samples in South Africans is not recommended.
\end{abstract}

Karen Charlton

karenc@uow.edu.au

1 School of Medicine, University of Wollongong, New South Wales, Australia and Illawarra Health and Medical Research Institute, Wollongong, NSW, Australia

2 South African MRC Developmental Pathways for Health Research Unit, School of Clinical Medicine, Faculty of Health Sciences, University of the Witwatersrand, Johannesburg, South Africa

3 Statistical Consultation Services, North-West University, 11 Hoffman Street, Potchefstroom; Private Bag X6001, Potchefstroom 2520, South Africa

4 Hypertension in Africa Research Team (HART), North-West University, Potchefstroom, South Africa

5 MRC Research Unit for Hypertension and Cardiovascular Disease, North-West University, Potchefstroom, South Africa

6 World Health Organization (WHO), Geneva, Switzerland

7 Chiang Mai University Research Institute for Health Sciences, Chiang Mai, Thailand

8 University of Newcastle Research Centre for Generational Health and Ageing, Newcastle, Australia

\section{Introduction}

It is widely accepted that excess dietary sodium or salt consumption is a major determinant of population blood pressure (BP) levels [1], contributing to the epidemic of hypertension and cardiovascular disease (CVD) [2]. Of the 56.9 million deaths worldwide in 2016, CVD including ischaemic heart disease and stroke were the world's biggest killers, accounting for over a quarter or 15.2 million deaths in that year alone [3]. Hypertension is estimated to be responsible for around half of these heart disease and stroke deaths, translating to 9.4 million deaths worldwide every year [4], and highlighting the need for prevention and treatment of raised BP to prevent these vascular events [5].

The Global Burden of Disease Study (1990-2016) suggests that, among individual dietary risk factors for CVD mortality and morbidity, a diet high in sodium contributed to $4.2 \%$ of deaths [6]. Alongside rapid economic, nutritional and demographic transition, low-to-middle-income countries (LMIC) are experiencing disproportionately rapid increases in hypertension prevalence [7, 8]. With many 
LMICs having no or inadequate universal health coverage [9], preventive public health strategies are urgently needed to reduce the subsequent cardiovascular morbidity and mortality.

An increasing number of countries are recognising the importance of population level salt reduction to reduce hypertension [10]. The WHO global targets to reduce noncommunicable disease (NCD) by 2025 [11] list a $30 \%$ relative reduction in mean population salt intake of as one of the seven targets defined in the framework. South Africa was the first country to implement legislation on mandatory maximum sodium levels in a wide range of processed foods, with a stepped implementation approach between 2016 and 2019 [12, 13]. Although the impact of the legislation has yet to be evaluated, estimates suggest salt intake should decrease by $0.85 \mathrm{~g} /$ person/day, resulting in an $11 \%$ reduction in annual CVD deaths and saving the government millions of dollars in health care costs [14, 15]. In many countries, sodium reduction in foods is not through legislation but voluntary reformulation by the food industry. For example, overall salt intake has reduced by $\sim 10 \%$ in the UK [16] owing to voluntary efforts by the food industry.

To evaluate the effectiveness of such country-level salt reduction strategies, reliable data are needed showing trends in population salt intake over time [17]. However, obtaining valid measures of population salt intake is challenging. Selfreported dietary data are often inaccurate as salt intake is both obvious (discretionary salt added to foods at the table or during cooking), as well as not-obvious, for example, in processed or packaged foods. Although a number of authors, including ourselves, have developed and validated short dietary assessment food frequency questionnaires to provide an estimate of salt intake that tend to correlate reasonably well with urinary biomarkers [18-21], such instruments tend to underestimate total $24 \mathrm{~h}$ urinary excretion.

In many high-income countries, including Australia, UK, and USA, most salt comes from processed foods. As such, it is possible to monitor changes to the salt content in the food supply as a proxy indicator for overall non-discretionary salt intake, although this requires food habits to be known $[12,22]$. However, estimating population salt intake from the food supply may also be inaccurate if discretionary salt intake is high, such as in China [23].

The gold standard method for measuring population salt intake is measurement of sodium excretion (as a proxy for intake) in 24-hour urine collections [24]. Although this method has been used to assess the age-specific, sex-specific, and region-specific average sodium and potassium intake' and its association with anthropometric characteristics in a sample of 1232 Italian adult hypertensives across 47 centres [25], it is onerous and often impractical for use in large surveys that aim to collect nationally representative data, especially in resource-poor LMICs [26]. Numerous authors have suggested that daily salt intake can be estimated from the sodium concentration in spot urine samples provided there is a measure of either the concentration or dilution of the urine. Other biochemical variables, such as albumin and catecholamines, are routinely measured in spot urine and expressed relative to urinary creatinine concentration, using creatinine concentration as a marker for diuresis [27]. However, the spot urine sodium/creatinine ratio has inherent limitations in estimating daily sodium excretion owing to day to day variability in both sodium and creatinine excretion [28, 29].

Various equations exist to estimate 24-hour sodium excretion from spot urine samples [30-33], many of which are based on 24-hour urinary creatinine estimates. These equations have mostly been developed in Japanese populations and there have been few applications of these equations in African populations [34]. The aim of this study was to investigate the validity of four such equations (INTERSALT [30], Tanaka [31], Kawasaki [32], Mage [33]) to predict 24-hour urinary Na excretion (24Una) from spot urine samples in South African adults.

\section{Methods}

\section{Study population and measures}

This analysis is based on data collected in a sub-study [35] of the World Health Organisation Study on global AGEing and adult health (WHO-SAGE, Wave 2). WHO-SAGE is a multinational longitudinal study examining the health and wellbeing of adult populations and the ageing process in over 42,000 respondents from six countries (China, Ghana, India, Mexico, Russia, and South Africa) [36]. SAGE South Africa Wave 2 data were collected in 2015 (August-December), following up households from Wave 1 (2007-2010) [37]. Spot and 24-hour urine collection was conducted in a nested subsample of Wave 2 respondents alongside the standard WHO-SAGE data collection (household, individual, and proxy questionnaires; anthropometry; dried blood spot collection; BP; and physical function tests $[36,38])$. The WHO/PAHO protocol was used for sodium determination in 24-hour urine [39], with the detailed protocol described previously [35]. In brief, respondents were requested to collect all urine produced for 24-hours, excluding the first pass urine on day 1, but including the first urine of the following morning (day 2) in a 5-litre plastic container containing $1 \mathrm{~g}$ thymol as preservative. The spot sample was collected without preservative from the second urine passed on day 1 (marking the start of the 24-hour collection), immediately aliquoted into three $15 \mathrm{ml}$ Porvair tubes (Porvair Sciences, 
Leatherhead, UK), then kept in a cool box powered by the fieldwork vehicles. The 24-hour sample was collected the next morning, volumes were recorded, and aliquots generated with all samples then shipped to the central laboratory in Durban, maintaining the cold chain. Sodium and potassium were determined using the indirect Ion Selective Electrode method and creatinine analysed using the standardised urinary Jaffe kinetic method (Beckman Coulter Synchron DXC600/800 System). Incomplete 24-hour urine collections were assumed and excluded from analyses if: total urinary volume $\leq 300 \mathrm{ml}$; or creatinine excretion $\leq 4$ $\mathrm{mmol} / \mathrm{day}$ (women) or $\leq 6 \mathrm{mmol} / \mathrm{day}$ (men) [40].

BP was measured by trained nurses using wrist-worn BP devices with positioning sensor (R6, Omron, Japan). Respondents had been seated for at least 5 minutes before three sequential measures were taken on the left arm (1 minute between each measure), with the wrist resting precisely at the level of the heart and the respondent seated with legs uncrossed. Such wrist BP devices have been shown to meet the validation criteria of the European Society of Hypertension International Protocol [41-43]. Hypertension was determined by systolic $\mathrm{BP} \geq 140 \mathrm{mmHg}$ or diastolic $\mathrm{BP} \geq 90 \mathrm{mmHg}$, or current antihypertensive medication use. Mean arterial pressure (MAP) was calculated using the formula: $\mathrm{MAP}=(\mathrm{SBP}-\mathrm{DBP}) \times 0.41+$ DBP [44].

All respondents provided written informed consent prior to taking part in the study. The study complied with the Declaration of Helsinki [45], with ethical approval from the WHO Ethics Review Committee [RPC149], the North-West University Health Research Ethics Committee (Potchefstroom, South Africa) and the University of the Witwatersrand Human Research Ethics Committee (Johannesburg, South Africa). Interviews were conducted in the respondents' home languages, with consent forms available in the most widely spoken languages for each region.

\section{Data capture and analysis}

An electronic data capture system was used during face-toface interviews. Stata/IC version 15.1 was used for statistical analyses. Categorical data are presented as frequencies and percentages. Data were checked for normality using the Skewness-Kurtosis test, with non-parametric data presented as medians and interquartile ranges and log transformation used for all non-normal outcomes. The following equations were evaluated to assess the accuracy of using spot urine samples to predict 24-hour urinary sodium excretion (24UNa): (1) the INTERSALT equation [30]; (2) the Tanaka equation $[31 ;$ (3) the Kawasaki equation $[32$; and (4) the Mage equation [33]. Refer to Table 1 for further details.

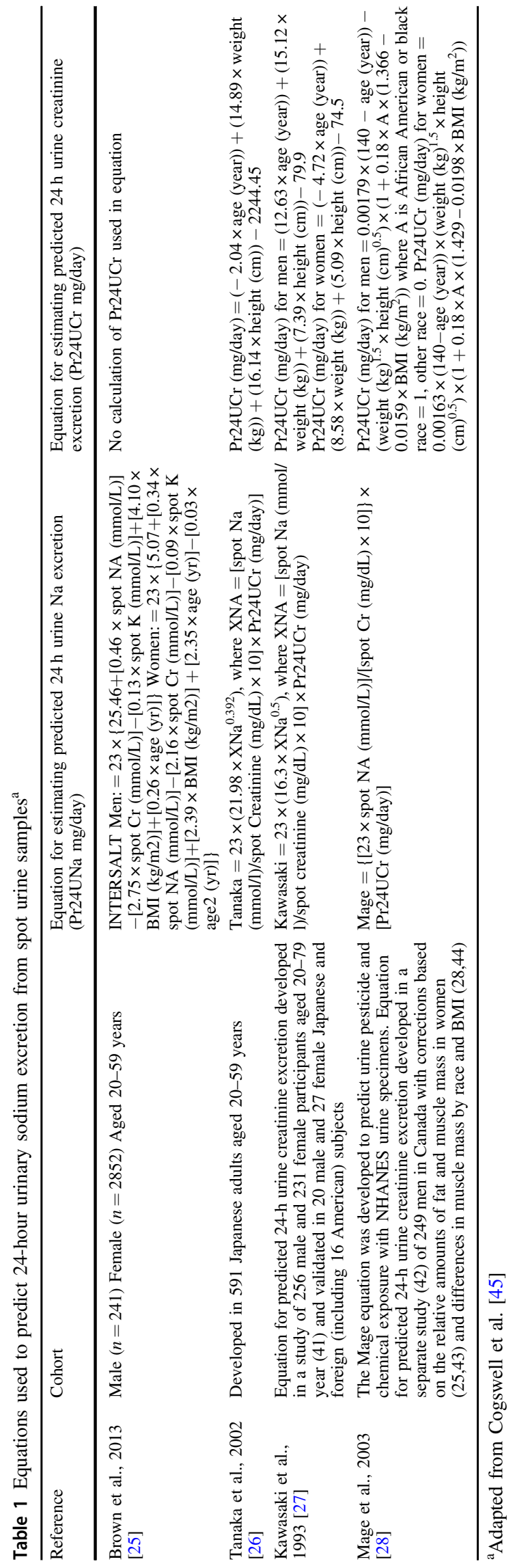


Spearman correlations were conducted to determine the association between observed 24UNa and the predicted 24hour urinary sodium excretion ( $\mathrm{Pr} 24 \mathrm{UNa}$ ) from each of the four equations with differences assessed using the Wilcoxon signed rank test. Agreement between 24Una and each Pr24UNa was assessed using Bland-Altman plots [46] and assessment of limits of agreement (LOA). Bland-Altman plots compare the mean difference (24UNa minus Pr24UNa) vs. the average of the two values. Both $24 \mathrm{UNa}$ and Pr24UNa values were transformed to their natural logarithms (ln) before analyses owing to the skewness in distributions. These are reported as the antilogarithm of the difference, i.e., the geometric mean of the $24 \mathrm{UNa} / \mathrm{Pr} 24 \mathrm{UNa}$ ratios and the antilogarithms of the LOA, which provide an interval within which $95 \%$ of the ratios lie. The LOA approach provides an informative analysis of reliability, including information about the magnitude of errors between the methods. The 95\% LOA represent a range of values within which $95 \%$ of all differences between methods are expected to fall. Using the standard deviation (sd) of differences between methods, the 95\% LOA were calculated for each of the four Pr24UNa values as mean agreement \pm 1.96 (sd diff). For example, mean agreement of $100 \%$ suggests exact agreement, whereas mean agreement of $80 \%$ indicates that the Pr24UNa overestimates observed $24 \mathrm{UNa}$ by $20 \%$, on average. All analyses were converted to daily salt $(\mathrm{NaCl})$ equivalents $(\mathrm{g} / \mathrm{d})$ for ease of interpretation and consistency of reporting against recommended cutoffs for optimal health [11]. Sensitivity and specificity of each of the equations were calculated to determine their ability to correctly classify participants according to the reference value of $5 \mathrm{~g}$ salt per day.

\section{Results}

Complete 24-hour urine collections were obtained from 889 of the participants included in the nested sample for urine collection of $n=1291$, representing $69 \%$ response rate. However, missing data reduced the sample size available for the present analysis to $n=438$. Reasons for missing data related to uploading issues of sociodemographic survey data (age, sex) and anthropometric (weight) and blood pressure measurements by the computer assisted personal interview system. Hypertension prevalence in the total survey sample $(n=1847)$ was $44 \%$ in older adults (50-plus years) and $25 \%$ in younger adults (18-49 years). The overall weighted prevalence of hypertension was $36 \%$ in both the total sample and the subsample, with no significant difference between these. Participant characteristics of the subsample are shown in Table 2. Based on analysis of complete 24-hour urine samples, sodium excretion varied widely with observed values of between 1 and $40 \mathrm{~g}$ salt/day, and a group median of $6.7 \mathrm{~g}$ salt/day (4.4-10.5). Median salt excretion was higher in the younger group (under 50 years of age; 8.5 (5.1-13.9) g salt/day) compared with the older group (50 years plus; $6.1(4.2-8.9) \mathrm{g}$ salt/day; $p<0.001)$. No significant differences were observed between men and women, or between respondents living in urban and rural areas.

Predicted urinary creatinine (Pr24UCr) was estimated according to the equations shown in Table 1 (excluding INTERSALT) and then entered into equations to estimate PrUNa.

There were significant differences (Wilcoxon signed rank test) between measured urinary creatinine $(24 \mathrm{UCr})$ and Pr24UCr (Table 3). Bland-Altman plots showed that all equations resulted in a systematic bias with higher observed creatinine values, resulting in a greater degree of underestimate in the group as a whole and in men and women independently (data not shown). Log transformation of the values did not remove this systematic bias. Neither BMI, waist-to-height ratio nor age could be used to predict those individuals with high measured daily creatinine excretion, for whom the predictive equations may be less accurate (data not shown). Table 3 shows the median difference between measured and predicted creatinine values, and the poor correlation between the measured value and all estimated values.

Investigating the median differences between the observed and predicted urinary sodium levels (Table 4) showed that the group estimates from the four equations were all significantly different to the measured value. Spearman correlations between 24UNa and Pr24UNa $(\mathrm{mmol} / \mathrm{L})$ were significant but weak for all equations. INTERSALT tended to underestimate sodium excretion, especially at lower levels, whereas Kawasaki and Mage overestimated 24UNa (Fig. 1).

The Bland-Altman curves suggest systematic bias for both INTERSALT and Tanaka equations. Geometric means (back transformed logarithmic data of differences) indicate: INTERSALT underestimates $24 \mathrm{UNa}$ by $306 \%$; Kawasaki overestimates by $43 \%$, whereas Tanaka performed best with a mean underestimation of $7 \%$. The Mage equation performed the worst, with a mean overestimation of $55 \%$ but unacceptably wide limits of agreement (LOA: $57 \%$ underestimation to $1321 \%$ overestimation). Clearly, this level of bias is outside the scope of an acceptable range [47].

The Tanaka, Kawasaki, and Mage equations had a high sensitivity (95.6-97.7\%) for correctly categorising individuals consuming more than the WHO recommendation of a maximum $5 \mathrm{~g}$ salt/day (Table 5), because of their bias to overestimate observed intakes. In contrast, specificity was very low (5-12\%), demonstrating their inability to correctly detect those individuals consuming $<5 \mathrm{~g}$ salt/d. 
Table 2 Sociodemographic and health characteristics of WHO-SAGE nested salt study sample, South Africa Wave 2 (2015)

\begin{tabular}{lccc}
\hline & Nested salt study $N=438$ & Male $n=106$ & Female $n=332$ \\
\hline Age, years & $55(40-65)$ & $52(37-63)$ & $56(43-65)$ \\
50 years and above, $n(\%)$ & $284(65)$ & $61(58)$ & $223(67)$ \\
Ethnicity, $n(\%)$ & & & \\
Black African & $319(73)$ & $78(74)$ & $241(73)$ \\
Coloured & $73(17)$ & $16(15)$ & $57(17)$ \\
Indian & $36(8)$ & $7(7)$ & $29(9)$ \\
White & $10(2)$ & $5(5)$ & $5(2)$ \\
Rural, $n(\%)$ & $129(30)$ & $30(28)$ & $99(30)$ \\
Education, years & $9(7-12)$ & $10(6-12)$ & $8(6-12)$ \\
BMI, kg/m & $25.6(22.3-29.7)$ & $30.4(25.7-35.6)$ \\
Waist-to-height ratio & $29.1(24.2-34.7)$ & $0.53(0.46-0.59)$ & $0.61(0.52-0.68)$ \\
Systolic BP, mmHg & $0.58(0.50-0.66)$ & $129(119-143)$ & $128(118-142)$ \\
Diastolic BP, mmHg & $129(118-142)$ & $82(74-89)$ & $80(72-88)$ \\
MAP, mmHg & $80(73-88)$ & $100(94-111)$ & $99(93-111)$ \\
Hypertensive, $n(\%)$ & $99(93-111)$ & $49(46)$ & $137(41)$ \\
AHT use, $n(\%)$ & $186(42)$ & $8(8)$ & $41(12)$ \\
Previous stroke, $n(\%)$ & $49(11)$ & $3(3.3)$ & $1(0.4)$ \\
Diabetic, $n(\%)$ & $4(1.2)$ & $7(8)$ & $28(11)$ \\
Current smoker, $n(\%)$ & $35(10)$ & $23(25)$ & $26(11)$ \\
Alcohol use, $n(\%)$ & $49(15)$ & $34(37)$ & $28(11)$ \\
\hline All & $62(18)$ & 25 (1) & \\
\hline
\end{tabular}

All data shown as median (IQR, interquartile range) unless otherwise indicated. Smokers identified by selfreport. Hypertensive categorised as $\mathrm{BP} \geq 140 / 90 \mathrm{mmHg}$ or current medication. $B M I$ body mass index, MAP mean arterial pressure, $A H T$ antihypertensive medication use within the last 2 weeks; alcohol use within the last month

Table 3 Comparison between measured 24-hour (24UCr) and predicted $24 \mathrm{~h}$ urinary creatinine excretion $(\operatorname{Pr} 24 \mathrm{UCr})$

\begin{tabular}{llcrcc}
\hline & Median (IQR) & Median difference & $\begin{array}{l}P \text { value of } \\
\text { difference }^{\text {a }}\end{array}$ & $\begin{array}{l}\text { Spearman } \\
\text { correlation } \\
\text { coefficient }\end{array}$ & $\begin{array}{l}\text { Spearman } \\
\text { correlation } \\
p \text { value }\end{array}$ \\
\hline $\begin{array}{l}\text { Measured 24UCr predicted } \\
\text { 24UCr using equations }\end{array}$ & $1094.3(784.6-1679.9)$ & & & & \\
Tanaka & $1257.6(1042.4-1496.7)$ & $-92.8(-495.6 ; 577.0)$ & 0.977 & 0.1512 & 0.0015 \\
Kawasaki & $1160.9(1006.9-1639.6)$ & $-163.7(-583.9 ; 365.2)$ & $<0.001$ & 0.1418 & 0.0029 \\
Mage & $1093.3(839.3-1422.9)$ & $59.9(-344.1 ; 649.3)$ & $<0.001$ & 0.1841 & 0.0001 \\
\hline
\end{tabular}

${ }^{a}$ Wilcoxon Signed Rank Test used to compare median values

INTERSALT showed $100 \%$ specificity but only $2 \%$ sensitivity, thus grossly underestimating observed salt intakes.

\section{Discussion}

This study assessed whether four previously published and commonly used equations could be used to estimate $24 \mathrm{UNa}$ (as a proxy for daily salt intake) using spot urine samples. The findings indicate that these equations are not appropriate for use in a South African adult population. Tanaka [31], Kawasaki [32], and Mage [33] equations estimate daily creatinine excretion as a proxy for urine 24-hour volume, and were developed in various populations using regression models incorporating age, weight, height, sex, and in some cases, ethnicity. Our findings suggest that both the creatinine and the sodium estimates from these equations are significantly different to measured 24-hour values. Tanaka, Kawasaki, and Mage all overestimated salt intake. In contrast, the INTERSALT equation [30], which does not calculate 24-hour creatinine, systematically underestimated salt intake.

Our findings are supported by previous work in Indian, white, and black subgroups in South Africa, also showing that Tanaka and Kawasaki equations overestimated sodium excretion [48], although that study suggested that 
Table 4 Summary of measured 24-hour and predicted 24-hour urinary $\mathrm{Na}$ excretion, expressed as salt equivalents ${ }^{\mathrm{a}}$ per day

\begin{tabular}{|c|c|c|c|c|c|}
\hline & Measured & INTERSALT & Tanaka & Kawasaki & Mage \\
\hline All, $n=438$ & $7.0(4.4-10.5)$ & $3.0(2.5-3.5)$ & $8.0(6.8-9.5)$ & $13.1(10.2-16.7)$ & $25.5(15.7-41.7)$ \\
\hline Men, $n=106$ & $7.7(4.6-11.2)$ & $3.7(3.1-4.2)$ & $8.1(6.9-9.1)$ & $18.5(14.8-22.3)$ & $25.9(16.6-42.5)$ \\
\hline Women, $n=332$ & $6.5(4.4-10.3)$ & $2.8(2.4-3.2)$ & $7.9(6.7-9.6)$ & $11.9(9.7-14.9)$ & $25.4(15.2-41.4)$ \\
\hline Black, $n=319$ & $6.4(4.4-10.6)$ & $3.0(0(2.6-3.5)$ & $8.0(6.8-9.5)$ & $13.2(10.2-16.7)$ & $27.0(16.7-43.1)$ \\
\hline Other ethnicity, $n=119$ & $7.5(4.3-10.3)$ & $2.8(2.4-3.4)$ & $8.0(6.5-9.7)$ & $12.9(9.8-16.5)$ & $22.2(12.0-35.0)$ \\
\hline 50 years and above, $n=284$ & $6.1(4.2-8.9)$ & $2.9(2.3-3.4)$ & $8.0(6.5-9.6)$ & $12.8(9.9-16.5)$ & $23.1(13.1-40.0)$ \\
\hline Below 50 years, $n=154$ & $8.5(5.1-13.9)$ & $3.1(2.8-3.5)$ & $8.0(6.9-9.2)$ & $13.7(10.5-16.8)$ & $30.7(20.6-44.2)$ \\
\hline Difference $^{\mathrm{b}}$ & & $3.77(1.64 ; 7.09)$ & $-1.28(-3.52 ; 1.97)$ & $-6.24(-9.45 ;-2.22)$ & $-17.18(-31.96 ;-8.42)$ \\
\hline$P$ values ${ }^{\mathrm{b}}$ & & $<0.001$ & 0.0118 & $<0.001$ & $<0.001$ \\
\hline
\end{tabular}

All data are shown as median (IQR, interquartile range)

a24-hour salt $(\mathrm{NaCl})$ equivalent $(\mathrm{g} /$ day $)=(24$-hour $\mathrm{Na}(\mathrm{mg} /$ day $) / 1000) \times 2.5$

${ }^{b}$ Difference is a summary variable of the differences between the measured and predicted 24-hour urinary salt excretion for each participant, assessed using the Wilcoxon Signed Rank Test
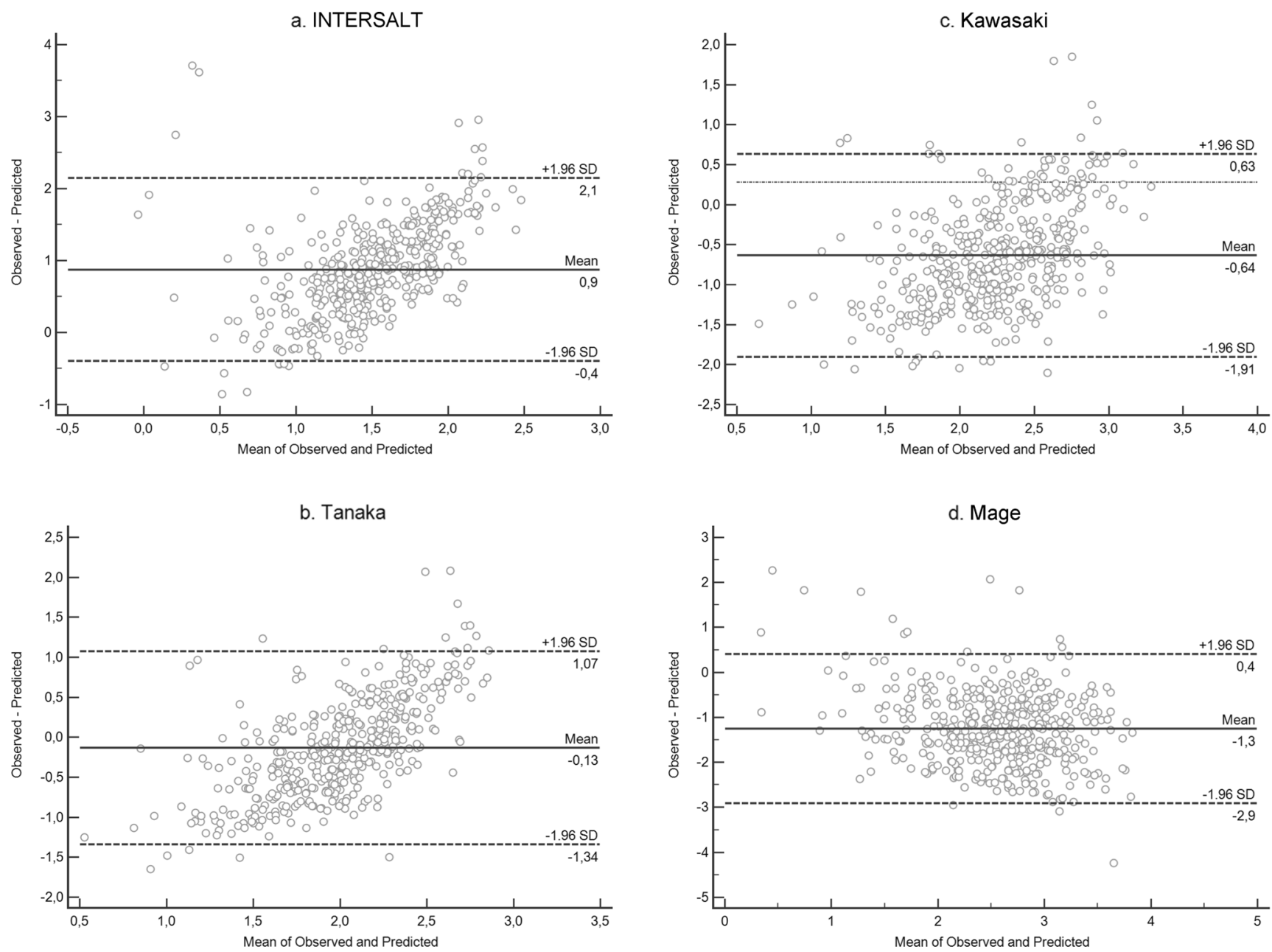

Fig. 1 Bland-Altman plots of $\log$ measured vs $\log$ predicted $24 \mathrm{~h}$ urinary sodium (mg/day). a INTERSALT equation. b Tanaka equation. b Kawasaki equation. d Mage equation

INTERSALT performed better, and it did not assess the Mage equation. In the current study, the degree of bias was considerable with all four equations, but smallest with the Tanaka equation. Likewise, in a sample of 554 participants (45-79 years; 58\% African American) from the MultiEthnic Study of Atherosclerosis (MESA) and the Coronary 
Table 5 Sensitivity and specificity of prediction equations vs observed 24-hour urinary salt

\begin{tabular}{|c|c|c|c|c|c|}
\hline \multirow[t]{2}{*}{ Equations } & \multirow{2}{*}{$\begin{array}{l}\text { Predicted salt intake } \\
\text { equivalent category }\end{array}$} & \multicolumn{2}{|c|}{ Measured (observed) salt intake equivalent category } & \multirow[t]{2}{*}{ Specificity $\%$} & \multirow[t]{2}{*}{ Sensitivity $\%$} \\
\hline & & Less than $5 \mathrm{~g} /$ day, $N=140$ & $\geq 5$ g/day, $N=298$ & & \\
\hline \multicolumn{6}{|c|}{ INTERSALT } \\
\hline \multicolumn{2}{|c|}{$<5$ g/day, $n=432$} & 140 & 292 & 100 & 2.0 \\
\hline \multicolumn{2}{|c|}{$\geq 5 \mathrm{~g} /$ day, $n=6$} & 0 & 6 & & \\
\hline \multicolumn{6}{|l|}{ Tanaka } \\
\hline \multicolumn{2}{|c|}{$<5$ g/day, $n=30$} & 17 & 13 & 12.1 & 95.6 \\
\hline \multicolumn{2}{|c|}{$\geq 5$ g/day, $n=408$} & 123 & 285 & & \\
\hline \multicolumn{6}{|l|}{ Kawasaki } \\
\hline \multicolumn{2}{|c|}{$<5 \mathrm{~g} /$ day, $n=12$} & 7 & 5 & 5.0 & 98.3 \\
\hline \multicolumn{2}{|c|}{$\geq 5$ g/day, $n=426$} & 133 & 293 & & \\
\hline \multicolumn{6}{|l|}{ Mage } \\
\hline \multicolumn{2}{|c|}{$<5$ g/day, $n=19$} & 12 & 7 & 8.6 & 97.7 \\
\hline \multicolumn{2}{|c|}{$\geq 5$ g/day, $n=419$} & 128 & 291 & & \\
\hline
\end{tabular}

Artery Risk Development in Young Adults (CARDIA) studies in the United States, the Tanaka equation produced the least bias overall in predicting 24-hour sodium excretion. However, the authors also reported that no single equation worked well across subgroups of sex and ethnicity [49]. We did not assess ethnicity- and sex-specific results in the current study as the majority of participants were African and the study was not powered to investigate sex differences.

Even when both the spot and 24-hour urine samples are collected from the same individuals on the same day, validation studies report large differences between estimated and measured 24-hour sodium excretion [50, 51]. The magnitude of bias seen in sodium excretion estimates from spot urine samples varies between different populations. For example, data from the prospective PURE study conducted in eleven LMICs ( $n=1083 ; 35-70$ years) found good correlation between measured and estimated sodium excretion in the total sample [50], but poor correlations in the Chinese cohort [51]. A further study in Britain and Italy found low agreement between estimated and measured 24UNa, again suggesting ethnic differences [52]. In Japan, the Kawasaki equation produced estimates from second morning void urines that correlated well (correlation coefficient 0.73) with measured values [32]. Although the Tanaka equation applied to random spot urines showed a lower correlation (correlation coefficient 0.54) in a Japanese population sample [31]. This may suggest that the second morning void (as used in our study) gives the best potential for assessing performance of these equations. However, in a US sample, correlation coefficients were moderate (0.4-0.6) for all prediction equations at various times of spot urine collection with Bland-Altman plots indicating significant over- and underestimation across low-to-high values of sodium excretion [53]. Furthermore, poor reproducibility indicates that estimation methods perform inconsistently. Our study found only weak correlations $(<0.22)$ for all four equations studied.

As highlighted in a recent editorial by Cappuccio and D'Elia [54], there is a need to estimate population salt intakes to support the monitoring and evaluation of population salt reduction initiatives while avoiding the burden of 24-hour urine collections. The WHO NCD Global Action Plan has set voluntary targets for maximum population level salt intakes at $5 \mathrm{~g}$ per day [11]. Our study suggests that monitoring of country-level progress toward realising this goal by 2025 cannot be achieved, at least in South Africa, using sodium estimates from spot urine samples.

Furthermore, the South African mandatory salt reduction policy (introduced in June 2016) is expected to result in average reductions in salt intake of $0.85 \mathrm{~g}$ per day [8, 9]. The unacceptably large magnitude of error associated with each of these prediction equations indicates their use would not accurately detect such reductions in salt intake. Indeed, the inability of most of the prediction equations to accurately classify individuals with salt intakes below $5 \mathrm{~g}$ per day means that incorrect assumptions could be made regarding the success of this salt reduction strategy. Our study is the first to systematically explore the utility of all four equations for use in monitoring the change in South Africa's population salt intake using a nationally representative sample.

The strengths of the study relate to the rigorous random selection procedures of the WHO-SAGE survey within which this study was nested. There are various limitations to the current analysis. Although 24-hour urine collection remains the gold standard for estimating population sodium intake, only a single 24-hour collection was taken as the 
standard against which to validate the prediction equations. Other research suggests that multiple consecutive 24-hour samples may be needed ( 3 days plus) to better estimate individual sodium intake [55]. Although this is clearly a challenge for any large survey, we are currently exploring methods to correct population single 24-hour collection using a smaller subsample of 3-day collections in Wave 3 in South Africa.

An additional limitation is the reduction in sample size from incomplete data. Complete 24-hour urine collections were achieved by $69 \%$ of the nested sample $(n=889$ of 1291), but other missing data reduced the sample size available for analysis to $n=438$. A further limitation is that the cut-off values used to assess completeness of 24-hour urine collection (24-hour volume $\geq 300 \mathrm{ml} /$ day; creatinine $\geq$ $4 \mathrm{mmol} /$ day (women) or $\geq 6 \mathrm{mmol} /$ day (men) [40]) may not be optimal for South African adults. For example, populations with low protein intakes exhibit more variability in daily creatinine excretion than well-nourished populations, with values often lower than $1 \mathrm{~g}(8.84 \mathrm{mmol})$ per day [56]. As such, it is possible our approach is conservative, though further research is needed to determine optimal thresholds against which to assess urine collection completeness. Another limitation of the study is that neither dietary protein intake, nor lean body mass were assessed. These two variables would inform the interpretation of low urinary creatinine concentrations, though it is well documented that women have lower excretion of creatinine than men and that creatinine excretion reduces with age [57, 58]. The current South African sample is older with higher levels of obesity than the populations in whom these equations were initially validated, and this may explain some of the differences observed. A final limitation was that the spot urine sample was not provided at the same time of day by all participants. Although some researchers suggest afternoon spot urine samples are best [59], the timing of the spot urine sample fails to offset bias and under- or overestimates resulting from the use of prediction equations [53]. Indeed, our results suggest that all of the methods assessed for estimating 24-hour creatinine and/or sodium excretion from a spot urine sample result in significant bias in an African population. A convenient method for estimating population sodium intake is urgently needed in the region, but accurate equations using spot urine samples are yet to be developed for use in South Africans.

\section{Conclusion}

Previously published and commonly used equations to predict daily salt intake from sodium concentrations in spot urine samples are inappropriate for use in an adult South African population. Further research is needed to understand why these equations perform so poorly, and to develop population specific methods to accurately assess sodium intake for large surveys. Based on our findings, the use of these prediction equations with spot urine samples to monitor changes in salt intake in South Africa is not recommended.

\section{Summary table}

\section{What is known about topic}

- Many countries have adopted salt-reduction targets in an attempt to curb hypertension.

- Accurate measurement of population-level salt intake is required to monitor progress toward salt-reduction targets.

- Analysis of $24 \mathrm{~h}$ urinary $\mathrm{Na}$ excretion is the gold standard measurement to estimate salt intake but this is burdensome.

- Prediction equations based on a casual (spot) urine collection have been developed.

- Validity of these equations for use in African populations remains to be demonstrated.

\section{What this study adds}

- In a nationally representative sample of South Africans, the Tanaka, Kawasaki, and Mage prediction equations overestimated $24 \mathrm{~h}$ urinary $\mathrm{Na}$ excretion.

- The Intersalt equation underestimated $24 \mathrm{~h}$ urinary $\mathrm{Na}$.

- All four equations had an unacceptably wide degree of bias and are not recommended for use in South Africans.

- Further research is needed to understand why these equations perform so poorly in this ethnic group.

- Population specific methods to accurately assess sodium intake for large surveys are required.

Acknowledgements We thank all study participants and acknowledge Dr. Stephen Rule, Dr. Robin Richards, and Mr. Godfrey Dlulane of Outsourced Insight who coordinated the data collection. Jordan Stanford and Ezinne Igwe are thanked for editorial assistance.

Funding This work is supported by an agreement with the CDC Foundation with financial support provided by Bloomberg Philanthropies, and a Partnerships \& Research Development Fund (PRDF) grant from the Australia Africa Universities Network. SAGE is supported by WHO and the Division of Behavioural and Social Research (BSR) at the National Institute on Aging (NIA), US National Institutes of Health, through Interagency Agreements (OGHA 04034785; YA1323-08-CN-0020; Y1-AG-1005-01) with WHO and a Research Project Grant R01AG034479.

Author contributions $\mathrm{KC}, \mathrm{PK}, \mathrm{NN}$ designed research; LJW implemented research; GC and MC performed analyses; KC, LJW, AES, 
MC, PK wrote the paper; KC had primary responsibility for final content. All authors read and approved the final manuscript. The Developmental Pathways for Health Research Unit acknowledges the South African Medical Research Council for support.

\section{Compliance with ethical standards}

Conflict of interest The authors declare that they have no conflict of interest.

Disclaimer and exclusive licence statement The content of this manuscript is solely the responsibility of the authors and does not necessarily represent the official views of the World Health Organization or the funding bodies. Professor Karen Charlton takes responsibility for the contents of this article.

Publisher's note: Springer Nature remains neutral with regard to jurisdictional claims in published maps and institutional affiliations.

\section{References}

1. Cappuccio FP. Cardiovascular and other effects of salt consumption. Kidney Int Suppl. 2013;3:312-5.

2. He FJ, Burnier M, MacGregor GA. Nutrition in cardiovascular disease: salt in hypertension and heart failure. Eur Heart J. 2011;32:3073-80.

3. World Health Organisation. The top 10 causes of death: WHO Press. 2018; http://www.who.int/en/news-room/fact-sheets/detail/ the-top-10-causes-of-death.

4. Lim SS, Vos T, Flaxman AD, Danaei G, Shibuya K, Adair-Rohani $\mathrm{H}$, et al. A comparative risk assessment of burden of disease and injury attributable to 67 risk factors and risk factor clusters in 21 regions, 1990-2010: a systematic analysis for the Global Burden of Disease Study 2010. Lancet. 2012;380:2224-60.

5. Yusuf S. Preventing vascular events due to elevated blood pressure. Circulation. 2006;113:2166-8.

6. Gakidou E, Afshin A, Abajobir AA, Abate KH, Abbafati C, Abbas KM, et al. Global, regional, and national comparative risk assessment of 84 behavioural, environmental and occupational, and metabolic risks or clusters of risks, 1990-2016: a systematic analysis for the Global Burden of Disease Study. Lancet. 2017;390:1345-422.

7. Forouzanfar MH, Liu P, Roth GA, Ng M, Biryukov S, Marczak L, et al. Global burden of hypertension and systolic blood pressure of at least 110 to $115 \mathrm{~mm} \mathrm{Hg}, 1990-2015$. JAMA. 2017;317:165-82.

8. Irazola VE, Gutierrez L, Bloomfield G, Carrillo-Larco RM, Dorairaj P, Gaziano T, et al. Hypertension prevalence, awareness, treatment, and control in selected LMIC communities: results from the NHLBI/UHG network of centers of excellence for chronic diseases. Glob heart. 2016;11:47-59.

9. Yusuf S, Rangarajan S, Teo K, Islam S, Li W, Liu L, et al. Cardiovascular risk and events in 17 low-, middle-, and highincome Countries. N Engl J Med. 2014;371:818-27.

10. Trieu K, Neal B, Hawkes C, Dunford E, Campbell N, RodriguezFernandez R, et al. Salt reduction initiatives around the world - a systematic review of progress towards the global target. PLoS ONE. 2015;10:e0130247.

11. World Health Organization. Global action plan for the prevention and control of noncommunicable diseases 2013-2020 Geneva: WHO Press, World Health Organization. 2013; http://africahea 1thforum.afro.who.int/IMG/pdf/global_action_plan_for_the_ prevention_and_control_of_ncds_2013-2020.pdf.
12. Charlton K, Webster J, Kowal P. To legislate or not to legislate? A comparison of the UK and South African approaches to the development and implementation of salt reduction programs. Nutrients. 2014;6:3672-95.

13. Prior RL, $\mathrm{Wu} X, \mathrm{Gu} \mathrm{L}$. Identification and urinary excretion of metabolites of 5-(hydroxymethyl)-2-furfural in human subjects following consumption of dried plums or dried plum juice. J Agric Food Chem. 2006;54:3744-9.

14. Watkins DA, Olson ZD, Verguet S, Nugent RA, Jamison DT. Cardiovascular disease and impoverishment averted due to a salt reduction policy in South Africa: an extended cost-effectiveness analysis. Health Policy Plan. 2016;31:75-82.

15. Bertram MY, Steyn K, Wentzel-Viljoen E, Tollman S, Hofman KJ. Reducing the sodium content of high-salt foods: effect on cardiovascular disease in South Africa. South Afr Med J. 2012;102:743-5.

16. Shankar B, Brambila-Macias J, Traill B, Mazzocchi M, Capacci S. An evaluation of the UK Food Standards Agency's salt campaign. Health Econ. 2013;22:243-50.

17. Popkin BM. Global nutrition dynamics: the world is shifting rapidly toward a diet linked with noncommunicable diseases. Am J Clin Nutr. 2006;84:289-98.

18. D'Elia L, Manfredi M, Strazzullo P, Galletti F. Validation of an easy questionnaire on the assessment of salt habit: the MINISALSIIA Study Program. Eur J Clin Nutr. 2019;73:793-800.

19. Charlton KE, Steyn K, Levitt NS, Jonathan D, Zulu JV, Nel JH. Development and validation of a short questionnaire to assess sodium intake. Public Health Nutr. 2008;11:83-94.

20. Ferreira-Sae M-CS, Gallani M-CB, Nadruz W, Rodrigues RC, Franchini KG, Cabral PC, et al. Reliability and validity of a semiquantitative FFQ for sodium intake in low-income and lowliteracy Brazilian hypertensive subjects. Public Health Nutr. 2009;12:2168-73.

21. Sasaki S, Ishihara J, Tsugane S. Validity of a self-administered food frequency questionnaire in the 5-year follow-up survey of the JPHC Study Cohort I to assess sodium and potassium intake: comparison with dietary records and 24-hour urinary excretion level. J Epidemiol. 2003;13:102-5.

22. Medina-Remón A, Tresserra-Rimbau A, Valderas-Martinez P, Estruch R, Lamuela-Raventos RM. Polyphenol Consumption and Blood Pressure. In: Watson RR, Preedy VR, Zibadi S, eds. Polyphenols in Human Health and Disease. San Diego: Academic Press; 2014. pp 971-87.

23. Mancia G, Oparil S, Whelton PK, McKee M, Dominiczak A, Luft FC, et al. The technical report on sodium intake and cardiovascular disease in low- and middle-income countries by the joint working group of the World Heart Federation, the European Society of Hypertension and the European Public Health Association. Eur Heart J. 2017;38:712-9.

24. Ji C, Sykes L, Paul C, Dary O, Legetic B, Campbell NR, et al. Systematic review of studies comparing 24-hour and spot urine collections for estimating population salt intake. Rev Panam de salud publica. 2012;32:307-15.

25. Galletti F, Agabiti-Rosei E, Bernini G, Boero R, Desideri G, Fallo $\mathrm{F}$, et al. Excess dietary sodium and inadequate potassium intake by hypertensive patients in Italy: results of the MINISAL-SIIA study program. J Hypertens. 2014;32:48-56.

26. O'Donnell M, Mente A, Yusuf S. Sodium intake and cardiovascular health. Circ Res. 2015;116:1046-57.

27. Garde AH, Hansen AM, Kristiansen J, Knudsen LE. Comparison of uncertainties related to standardization of urine samples with volume and creatinine concentration. Ann Occup Hyg. 2004;48:171-9.

28. Rakova N, Juttner K, Dahlmann A, Schroder A, Linz P, Kopp C, et al. Long-term space flight simulation reveals infradian rhythmicity in human $\mathrm{Na}(+)$ balance. Cell Metab. 2013;17:125-31. 
29. Knuiman JT, Hautvast JG, van der Heijden L, Geboers J, Joossens $\mathrm{JV}$, Tornqvist $\mathrm{H}$, et al. A multi-centre study on within-person variability in the urinary excretion of sodium, potassium, calcium, magnesium and creatinine in 8 European centres. Hum Nutr Clin Nutr. 1986;40:343-8.

30. Brown IJ, Dyer AR, Chan Q, Cogswell ME, Ueshima H, Stamler $\mathrm{J}$, et al. Estimating 24-hour urinary sodium excretion from casual urinary sodium concentrations in Western populations: the INTERSALT study. Am J Epidemiol. 2013;177:1180-92.

31. Tanaka T, Okamura T, Miura K, Kadowaki T, Ueshima H, Nakagawa H, et al. A simple method to estimate populational 24-h urinary sodium and potassium excretion using a casual urine specimen. J Hum Hypertens. 2002;16:97-103.

32. Kawasaki T, Itoh K, Uezono K, Sasaki H. A simple method for estimating $24 \mathrm{~h}$ urinary sodium and potassium excretion from second morning voiding urine specimen in adults. Clin Exp Pharmacol Physiol. 1993;20:7-14.

33. Mage DT, Allen RH, Kodali A. Creatinine corrections for estimating children's and adult's pesticide intake doses in equilibrium with urinary pesticide and creatinine concentrations. J Expo Sci Environ Epidemiol. 2008;18:360-8.

34. Mizehoun-Adissoda C, Houehanou C, Chianea T, Dalmay F, Bigot A, Preux PM, et al. Estimation of daily sodium and potassium excretion using spot urine and 24-hour urine samples in a black population (Benin). J Clin Hypertens (Greenwich). 2016;18:634-40.

35. Charlton K, Ware LJ, Menyanu E, Biritwum RB, Naidoo N, Pieterse $\mathrm{C}$, et al. Leveraging ongoing research to evaluate the health impacts of South Africa's salt reduction strategy: a prospective nested cohort within the WHO-SAGE multicountry, longitudinal study. BMJ Open. 2016;6:11.

36. Kowal P, Chatterji S, Naidoo N, Biritwum R, Fan W, Lopez Ridaura R, et al. Data resource profile: the World Health Organization Study on global AGEing and adult health (SAGE). Int $\mathbf{J}$ Epidemiol. 2012;41:1639-49.

37. Phaswana-Mafuya N P, K, Schneider M, Makiwane M, Zuma K, Ramlagan S, Tabane C, et al. Study of Global Ageing and Adult Health (SAGE), South Africa 2007-2008. Geneva: WHO Press: World Health Organization. 2011; http://repository.hsrc.ac.za/ bitstream/handle/20.500.11910/3424/7255.pdf?sequence $=$ $1 \&$ isAllowed $=\mathrm{y}$.

38. Peltzer K, Phaswana-Mafuya N. Hypertension and associated factors in older adults in South Africa. Cardiovasc J Afr. 2013;24:66-72.

39. WHO/PAHO Regional Expert Group for Cardiovascular Disease Prevention through Population-wide Dietary Salt Reduction. Protocol for population level sodium determination in 24-hour urine samples Geneva: WHO Press: World Health Organization. 2010; https://www.paho.org/hq/dmdocuments/2013/24h-urineProtocol-eng.pdf.

40. Stolarz-Skrzypek K, Kuznetsova T, Thijs L, Tikhonoff V, Seidlerova J, Richart T, et al. Fatal and nonfatal outcomes, incidence of hypertension, and blood pressure changes in relation to urinary sodium excretion. JAMA. 2011;305:1777-85.

41. O’Brien E, Pickering T, Asmar R, Myers M, Parati G, Staessen J, et al. Working Group on Blood Pressure Monitoring of the European Society of Hypertension International Protocol for validation of blood pressure measuring devices in adults. Blood Press Monit. 2002;7:3-17.

42. Topouchian JA, El Assaad MA, Orobinskaia LV, El Feghali RN, Asmar RG. Validation of two automatic devices for selfmeasurement of blood pressure according to the International Protocol of the European Society of Hypertension: the Omron M6
(HEM-7001-E) and the Omron R7 (HEM 637-IT). Blood Press Monit. 2006;11:165-71.

43. Takahashi H, Yoshika M, Yokoi T. Validation of Omron RS8, RS6, and RS3 home blood pressure monitoring devices, in accordance with the European Society of Hypertension International Protocol revision 2010. Vasc Health Risk Manag. 2013;9:265-72.

44. Jilek J, Stork M, editors. Wrist cuff method determines mean arterial pressure in dual-cuff blood pressure system. International Conference on Applied Electronics; 9-10 Sept 2014.

45. World Medical Association. World Medical Association Declaration of Helsinki: ethical principles for medical research involving human subjects. JAMA. 2013;310:2191-4.

46. Bland JM, Altman DG. Measuring agreement in method comparison studies. Stat Methods Med Res. 1999;8:135-60.

47. Batterham MJ, Van Loo C, Charlton KE, Cliff DP, Okely AD. Improved interpretation of studies comparing methods of dietary assessment: combining equivalence testing with the limits of agreement. Br J Nutr. 2016;115:1273-80.

48. Swanepoel B, Schutte AE, Cockeran M, Steyn K, WentzelViljoen E. Monitoring the South African population's salt intake: spot urine v. 24 h urine. Public Health Nutr. 2018;21:480-8.

49. Allen NB, Zhao L, Loria CM, Van Horn L, Wang CY, Pfeiffer $\mathrm{CM}$, et al. The validity of predictive equations to estimate 24-hour sodium excretion: the MESA and CARDIA Urinary Sodium Study. Am J Epidemiol. 2017;186:149-59.

50. Mente A, O'Donnell MJ, Dagenais G, Wielgosz A, Lear SA, McQueen MJ, et al. Validation and comparison of three formulae to estimate sodium and potassium excretion from a single morning fasting urine compared to 24-h measures in 11 countries. J Hypertens. 2014;32:1005-14. discussion 15

51. Peng Y, Li W, Wang Y, Chen H, Bo J, Wang X, et al. Validation and assessment of three methods to estimate 24-h urinary sodium excretion from spot urine samples in chinese adults. PLoS ONE. 2016;11:e0149655.

52. Ji C, Miller MA, Venezia A, Strazzullo P, Cappuccio FP. Comparisons of spot vs 24-h urine samples for estimating population salt intake: validation study in two independent samples of adults in Britain and Italy. Nutr Metab Cardiovasc Dis. 2014;24:140-7.

53. Cogswell ME, Wang CY, Chen TC, Pfeiffer CM, Elliott P, Gillespie CD, et al. Validity of predictive equations for 24-h urinary sodium excretion in adults aged 18-39 y. Am J Clin Nutr. 2013;98:1502-13.

54. Cappuccio FP, Elia L. Evaluating population salt reduction programmes worldwide: the risk of cutting corners! Public Health Nutr. 2017;21:1-3.

55. Rakova N, Jüttner K, Rauh M, Dahlmann A, Goller U, Beck L, et al. Ultra long-term sodium balance studies during the Mars500 campaign. Aktuel- Ernahr. 2012;37:P9_5.

56. Bourdoux P. Evaluation of the iodine intake: problems of the iodine/creatinine ratio--comparison with iodine excretion and daily fluctuations of iodine concentration. Exp Clin Endocrinol Diabetes. 1998;106:S17-20.

57. Baxmann AC, Ahmed MS, Marques NC, Menon VB, Pereira AB, Kirsztajn GM, et al. Influence of muscle mass and physical activity on serum and urinary creatinine and serum cystatin $\mathrm{C}$. Clin J Am Soc Nephrol. 2008;3:348-54.

58. Barr DB, Wilder LC, Caudill SP, Gonzalez AJ, Needham LL, Pirkle JL. Urinary creatinine concentrations in the U.S. population: implications for urinary biologic monitoring measurements. Environ Health Perspect. 2005;113:192-200.

59. Mann SJ, Gerber LM. Estimation of 24-hour sodium excretion from spot urine samples. J Clin Hypertens 2010;12:174-80. 